\title{
The prevalence and impact of brucellosis in patients with hepatitis delta virus infection: inside the Brucella outbreak with cirrhosis
}

\author{
Burak Suvak ${ }^{1}$, Ahmet Cumhur Dulger ${ }^{1}$, Ozlem Suvak², Aysun Özel Yesilyurt ${ }^{3}$, Bilge Gultepe ${ }^{4}$, \\ Huseyin Guducuoglu ${ }^{5}$
}

\begin{abstract}
${ }^{1}$ Gastroenterology Department, Medical School, Yuzuncu Yıl University, Van, Turkey ${ }^{2}$ Department of Family Medicine, Kecioren Family Medicine Center, Ankara, Turkey ${ }^{3}$ Internal Medicine Department, Medical School, Yuzuncu Yıl University, Van, Turkey ${ }^{4}$ Microbiology Department, Vakıf Gureba Hospital, Istanbul, Turkey

${ }^{5}$ Microbiology Department, Yuzuncu Yıl University, Van, Turkey
\end{abstract}

Submitted: 19 December 2014

Accepted: 11 May 2015

Arch Med Sci 2017; 13, 2: 377-382

DOI: https://doi.org/10.5114/aoms.2017.65317

Copyright @ 2017 Termedia \& Banach

\begin{abstract}
Introduction: Hepatitis D virus (HDV) infection is a serious health problem leading to cirrhosis and hepatocellular carcinoma (HCC). Despite evidence that zoonotic infections are associated with end-stage liver disease, brucellosis in patients with delta hepatitis related to liver disease has not been well characterized. So, we examined this relationship using recent hospital-based data.

Material and methods: We analyzed data from 96 delta hepatitis patients (mean age: $52.5 \pm 12.8$ years; 50 male; 52 cirrhotics) and 117 (mean age: $50.4 \pm 7$ years; 60 male) control subjects who were selected from patients with splenomegaly. The Brucella Wright test in connection with blood culture was used to detect active Brucella infection. Demographic features, laboratory data, results of ultrasonographic examination of the abdomen and Wright agglutination titers were compared between groups.

Results: There were 9 (9\%) patients with active brucellosis in delta hepatitis patients. Compared to the control group, there was a statistically significant difference between groups in terms of having active brucellosis (9 vs. 2 patients; $p<0.001)$. Higher MELD scores were also associated with active Brucella infection $(p<0.005)$.

Conclusions: Patients with chronic hepatitis D related cirrhosis (CHD-C) were at risk of developing brucellosis requiring hospitalization. Higher Wright titers among patients with more advanced liver disease may reflect a unique phenomenon that requires further investigation to determine underlying causative factors.
\end{abstract}

Key words: brucellosis, cirrhosis, delta hepatitis.

\section{Introduction}

Hepatitis D virus (HDV) is a small, defective hepatotrophic RNA virus which requires the help of hepatitis $B$ virus (HBV) for its replication. It has been shown that chronic HBV and HDV co-infection causes more severe liver damage than in the case of chronic HBV infection alone [1]. Hepatitis $D$ virus is endemic in the Mediterranean basin, e.g. Turkey and Egypt

\author{
Corresponding author: \\ Burak Suvak Assist. Prof. \\ Gastroenterology Department \\ Medical Faculty \\ Yuzuncu Yil University \\ 65100 Van, Turkey \\ Phone: +90 4322251024 \\ E-mail: drsuvak@gmail.com
}


$[2,3]$. Delta hepatitis affects approximately $20 \%$ of individuals with HBV infection in the eastern part of Turkey, ultimately leading to fibrosis, cirrhosis and hepatocellular carcinoma HCC [4].

Human brucellosis is a zoonosis caused by facultative, non-motile, intracellular Gram-negative bacteria of the genus Brucella. Brucellosis is also an emerging zoonotic infection in developing countries and carries significant morbidity and mortality [5].

Distribution of emerging zoonoses has changed in the world over the past century because of improvements of sanitation and pasteurization techniques, coupled with economic advances [6]. Although infection rates have been declining in the western world, less is known about brucellosis prevalence and risk factors among patients with chronic hepatitis $D$ related cirrhosis (CHD-C). Human brucellosis is also an endemic disease in the middle east as well as in Turkey [7]. The lack of original studies to elucidate the connection between HDV and brucellosis has been a major obstacle in understanding the pathophysiological mechanisms between these diseases. It is a well known fact that liver cirrhosis has a high risk of bacterial infections [8]. For the identification of effects of brucellosis on CHD-C we examined the clinical and laboratory features of the patients. The objectives of this study were to examine the connection between human brucellosis and CHD-C. Another aim of this study was to investigate serum Wright agglutination titers in different Model for End-Stage Liver Disease (MELD) scores of the patients with CHD-C.

\section{Material and methods}

We analyzed data from adult delta hepatitis patients who were admitted to our gastroenterology clinic from June 2013 to June 2014. Baseline characteristics of the patients are shown in Table I. There were 96 patients (mean age: $52.5 \pm 12.8$ years; 50 male) with HDV, of whom 52 (54\%) had biopsy-proven cirrhosis. Exclusion criteria included prior liver transplantation, presence of hepatocellular carcinoma, and chronic renal insufficiency (creatinine level > $2.0 \mathrm{mg}$ per deciliter). The control group (117 subjects; mean age: $50.4 \pm 7$ years; 60 male) was selected from subjects who were evaluated because of splenomegaly. Data extraction was performed retrospectively. Demographics, laboratory data, results of ultrasonographic examination of the liver and Wright agglutination titers were compared between groups. MELD scores of the cirrhotic patients were assessed by_www.mayoclinic.org/.../meld-model-unos-mod. and were compared according to Brucella status. Characteristic features of delta hepatitis patients according to presence of brucellosis are shown in Table II. All patients with delta hepatitis underwent conventional liver ultrasonography. All patients or their legally authorized representatives provided written informed consent.

\section{Serologic studies}

Results of the samples that have HBsAg reactive anti-HDV antibodies micro-ELISA (Enzyme-Linked Immuno Sorbent Assay) (Triturus ELISA) with Dia. Pro Diagnostic Bioprobes anti-HDV IgG, Italy) kits were determined. The Wright agglutination test

Table I. Baseline characteristics of patients

\begin{tabular}{|c|c|c|c|c|}
\hline \multirow[t]{2}{*}{ Variable } & \multicolumn{2}{|c|}{$\begin{array}{l}\text { Non-cirrhotic delta hepatitis patients } \\
\qquad(n=44)\end{array}$} & \multicolumn{2}{|c|}{$\begin{array}{l}\text { Cirrhotic delta hepatitis patients } \\
\qquad(n=52)\end{array}$} \\
\hline & Mean & Standard deviation & Mean & Standard deviation \\
\hline Age [years] & 51.7 & 12.6 & 53.2 & 30 \\
\hline $\begin{array}{l}\text { Wright agglutination } \\
\text { titer }\end{array}$ & 30 & 115.7 & 97.6 & 43 \\
\hline WBC $\left[10^{3} / \mu l\right]$ & 7329 & 3808 & 6869 & 1920 \\
\hline $\mathrm{Hb}[\mathrm{g} / \mathrm{dl}]$ & 14.7 & 1.54 & 14.5 & 6.3 \\
\hline Plt $[\mu l]$ & 200.045 & 76897 & 122076 & 59334 \\
\hline AST $[U / I]$ & 51 & 43.6 & 72 & 62.6 \\
\hline ALT [U/l] & 60 & 56.7 & 70 & 68.7 \\
\hline Albumin [g/dl] & 3.94 & 0.51 & 3.75 & 0.73 \\
\hline Globulin [g/dl] & 3.53 & 0.64 & 3.70 & 3.25 \\
\hline MELD score & & & 9.46 & \\
\hline
\end{tabular}

WBC - white blood cells, Hb - hemoglobin, Plt - platelets, AST - aspartate aminotransferase, ALT - alanine aminotransferase, MELD Model For End-Stage Liver Disease. 
The prevalence and impact of brucellosis in patients with hepatitis delta virus infection: inside the Brucella outbreak with cirrhosis

Table II. Characteristic features of delta hepatitis patients according to presence of brucellosis

\begin{tabular}{|c|c|c|c|c|c|c|c|c|c|}
\hline \multirow[t]{2}{*}{ Variable } & \multicolumn{4}{|c|}{ Delta hepatitis without brucellosis } & \multicolumn{4}{|c|}{ Delta hepatitis with brucellosis } & \multirow[t]{2}{*}{$P$-value } \\
\hline & Mean & St. dev. & Min. & Max. & Mean & St. dev. & Min. & Max. & \\
\hline Age [years] & 51.2 & 12.1 & 20 & 80 & 60 & 15.5 & 27 & 78 & 0.027 \\
\hline $\mathrm{WBC}\left[10^{3} / \mu \mathrm{l}\right]$ & 7241 & 4337 & 2600 & 25200 & 6207 & 2131 & 4000 & 11800 & 0.638 \\
\hline $\mathrm{Hb}[\mathrm{g} / \mathrm{dl}]$ & 14.7 & 1.6 & 9.2 & 17.6 & 14.9 & 2.3 & 10.3 & 17.4 & 0.313 \\
\hline Plt $[\mu l]$ & 162560 & 80545 & 51000 & 356000 & 132307 & 72198 & 38000 & 265000 & 0.207 \\
\hline AST $[U / I]$ & 55 & 38 & 14 & 210 & 109 & 100 & 18 & 387 & 0.027 \\
\hline ALT [U/I] & 60 & 48 & 7 & 336 & 103 & 103 & 15 & 402 & 0.123 \\
\hline Albumin [g/dl] & 3.9 & 0.63 & 2.4 & 5.0 & 3.6 & 0.62 & 2.8 & 4.7 & 0.090 \\
\hline Globulin [g/dl] & 3.6 & 0.74 & 1.8 & 5.3 & 3.9 & 1.0 & 2.0 & 5.2 & 0.361 \\
\hline MELD score & 8.4 & 2.9 & 6.0 & 18.0 & 9.8 & 3.4 & 6.0 & 16.0 & 0.122 \\
\hline
\end{tabular}

WBC - white blood cells, $\mathrm{Hb}$ - hemoglobin, Plt - platelets, AST - aspartate aminotransferase, ALT - alanine aminotransferase, MELD Model For End-Stage Liver Disease.

was performed with U-shaped microtiter plates instead of tubes. Eight wells were used for each sample. The first well of $0.9 \mathrm{ml}$ was placed in saline diluent, while others were placed in $0.5 \mathrm{ml}$ of saline. Firstly $0.1 \mathrm{ml}$ of the sample was added to the first well to complete $1 \mathrm{ml}$; later $0.5 \mathrm{ml}$ of this mixture was added to the second well and $0.5 \mathrm{ml}$ of the second mixture was added to the third well and this process was continued to the tenth well. Then these dilutions were doubled by adding $50 \mu \mathrm{l}$ of standardized Brucella abortus (Spinreact, Spain) diluted in normal saline. Wright agglutination test reaction was performed after 24 -h incubation at $37^{\circ} \mathrm{C}$. Values of $1 / 160$ and above were considered negative. $1 / 40$ and $1 / 80$ as a result of the Brucellacapt agglutination is considered as a questionable negative test. Brucellacapt agglutination is similar to that obtained with the Coombs test, determining antibodies of all classes specific to Brucella. The Brucellacapt test (Vircell SL) was performed according to the manufacturer's instructions. Briefly, $50 \mu \mathrm{l}$ samples of serum dilutions were added to wells of a U-bottom microtiter plate coated with anti-total human immunoglobulin. Then $50 \mu \mathrm{l}$ of an antigen suspension (colored B. melitensis bacteria killed by formaldehyde treatment) was added to all the wells. The plates were sealed with adhesive tape and incubated at $37^{\circ} \mathrm{C}$ for $24 \mathrm{~h}$ in a dark humid chamber. Positive reactions show agglutination over the bottom of the well. Negative reactions are indicated by a pellet at the center of the bottom of the well [9]. A Wright serum agglutination test at a titer equal to or higher than $1: 160$ and a positive blood culture test in connection with a compatible clinical picture were considered as acute Brucella infection.

Past infection of brucellosis was defined as a Wright agglutination titer lower than $1 / 80$, a negative blood culture for brucellosis and lack of acute brucellosis-related symptoms including nocturnal fever, splenomegaly and leucopenia.

\section{Statistical analysis}

The data obtained from the hospital records were entered into SAS (SAS Institute, Release 9.4, 2014, Cary, NC) software for data analyses. Descriptive statistics of data were expressed with average \pm standard deviation, median and as a percentage using PROC MEANS and PROC FREQ in SAS. Pearson's $\chi^{2}$, likelihood chi square and Fisher's exact test were used for the independence test between qualitative variables. PROC TTEST for Student's $t$ test was used to evaluate the differences between means of cirrhotic and non-cirrhotic patients for normally distributed dependent variables. Mann-Whitney $U$ test by using PROC NPAR1WAY was performed to compare the differences between the median of cirrhotic and non-cirrhotic patients for non-normally distributed dependent variables. Differences were considered significant at $p<0.05$ level.

\section{Results}

There were no statistically significant differences between delta hepatitis patients and control subjects in age, gender or residential area profile. In 117 subjects of the control group, there were only $2(1.7 \%)$ patients with active brucellosis. On the other hand, 6 (5.1\%) patients had evidence of past Brucella infection in the control group. In 52 patients with CHD-C, there were 7 (13\%) confirmed active brucellosis cases and 3 (5.5\%) cases of past infection. Among 44 non-cirrhotic delta hepatitis patients, 2 (4.5\%) patients had active brucellosis and 2 (4.5\%) had evidence of 
past infection. There was a significant difference between the control group and delta hepatitis-related cirrhosis in terms of active brucellosis ( 2 vs. 7 patients; $p<0.005$ ). In the cirrhotic group, mean titer of the Wright agglutination test was $98 \pm 43$ and the mean MELD score was $9.5 \pm 3$.2. The mean titer of the Wright agglutination test among non-cirrhotic patients with delta hepatitis was $30 \pm 115$. There was a significant difference between groups in terms of the mean titers of the Wright agglutination test $(p<0.005)$. Furthermore, higher MELD scores were significantly associated with active brucellosis in cirrhotic delta hepatitis patients $(p<0.005)$.

Additionally, in cirrhotic delta hepatitis patients, there was a positive correlation between MELD scores and Brucella Wright test titers ( $p<$ 0.005). In cirrhotic patients, older age, higher aspartate aminotransferase (AST) levels and low levels of albumin were significantly associated with active brucellosis compared to cirrhotic patients who had no active brucellosis ( $60 \pm 15.5$ vs. 51.2 $\pm 12 ; 109.9 \pm 100$ vs. $55 \pm 38$; $3.6 \pm 0.64$ vs. $4 \pm 0.5$; $p=0.027 ; p=0.026$ and $p=0.041$ retrospectively). Other laboratory parameters among patients with liver cirrhosis were not statistically significant in terms of active brucellosis (all $p>0.05$ ). Brucella status among study subjects is shown in Figure 1.

\section{Discussion}

In this study, we demonstrated that the rate of active brucellosis was extraordinarily high among CHD-related cirrhosis. Delta hepatitis is a progressive liver disease caused by a defective HDV, which is a prevalent problem within the Mediterranean basin and middle east [2, 3, 10]. Furthermore, delta hepatitis treatment has not been established over the last decade, and both classical and pe-

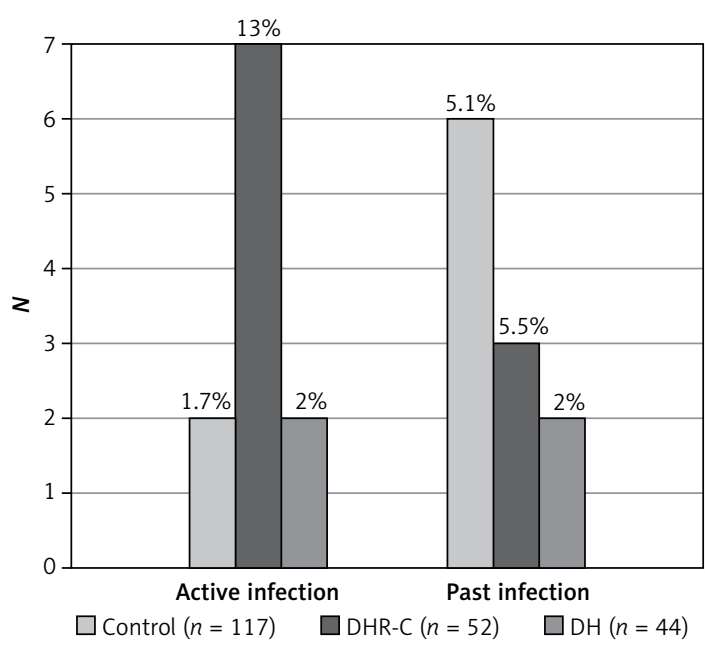

Figure 1. Brucella status among study subjects DHR-C - delta hepatitis related cirrhosis, DH - delta hepatitis. gylated interferons are treatment options for HDV infection. Patients with delta hepatitis can be treated with an interferon alpha based therapy if decompensated cirrhosis has been excluded [11]. Hepatitis D virus prevalence in the general Turkish population is $1.6 \%$, but data in Turkey are limited. However, in Turkey, there are few nationally representative data on the contribution of HDV infection to cirrhosis. On the other hand, the eastern part of the country is still home to one of the world's largest delta hepatitis populations. It is currently estimated that $20 \%$ of hepatitis B patients in the eastern part of Turkey are complicated by HDV infection, corresponding to approximately 400000 patients [12].

Brucellosis is a zoonotic infectious disease. It is caused by a small, non-motile, Gram-negative coccobacillus of the genus Brucella (B. melitensis, $B$. abortus, B. suis, and B. canis) and usually presents as recurrent fevers, fatigue and splenomegaly [13]. Major risk factors for human brucellosis include consumption of non-pasteurized milk products and close contact with infected animals. The diagnosis can be made by isolation of the organism from a blood culture and established by serologic testing using ELISA in connection with clinical findings as well as a history of consumption of raw milk products in an endemic area. Mediterranean countries including Turkey are high prevalence areas for human brucellosis [5]. The majority of Turkish cases have been reported from the eastern part of the country where consumption of traditionally produced unpasteurized milk-based cheese is common [14]. The Brucella Wright agglutination test is a simple method to screen Brucella infection. Despite its emerging importance, it is cumbersome, produces variable yields and has limited utility as a practical laboratory method [15]. We therefore used blood cultures for diagnosia of acute brucellosis. In our study, there were 17 patients with positive blood culture for Brucella. Cirrhotic patients are predisposed to bacterial infections that cause morbidity and mortality. One of the most important key concerns in cirrhotic patients is bacterial infections. While diminished hepatic function is clearly implicated in bacterial infections, its precise mechanisms remain unclear. Only Child-Pugh stage $C$ is determined as the most important factor to have bacterial infections $[16,17]$. It has also been reported that patients with advanced liver disease should be monitored closely for infections [18]. Nowadays, there is little information in the English literature regarding the impact of brucellosis in patients with cirrhosis due to HDV infection. Furthermore, there are no published data on the rate of Brucella infection in this unique patient population [18-20]. In the current study, active brucellosis rates were high among CHD-C patients overall (13\%), especially in 
comparison to healthy subjects, but even higher for those with higher MELD scores. Furthermore, we found that patients with advanced stages of cirrhosis also have significantly elevated levels of Brucella Wright test titers. These data show that impaired immunity in advanced stages of cirrhosis may contribute to this unique phenomenon. It is expected that the new era of immunology will provide much needed tools in this regard.

In terms of active brucellosis, our study showed that there were no differences between non-cirrhotic patients and the control group, while significant differences existed in the cirrhotic group. Since the control group was selected from subjects evaluated for splenomegaly of unknown origin (other than brucellosis), this condition could be associated with higher risks for infection. However, a higher rate of active brucellosis was detected in patients with cirrhosis compared to the control group selected with splenomegaly. The high prevalence of brucellosis in delta cirrhosis was also probably reflective of the higher prevalence of brucellosis in their residential areas. Brucellosis is an important health problem especially in endemic areas including the middle east [21-24]. In the current study, higher rates of brucellosis were attributed to poor hygienic conditions and health system barriers in the eastern part of the country. These poor hygienic conditions including lack of pasteurization are compounded in medically underserved communities with high rates of HDV infection.

It is a well-known fact that acute brucellosis may cause elevated levels of liver enzymes, mostly due to reticuloendothelial system involvement of the liver. On the other hand, elevated levels of AST and hypoalbuminemia are mostly detected in patients with cirrhosis at an advanced stage. In our study, cirrhotic patients who also had active brucellosis had higher levels of AST and higher age compared to Brucella-free counterparts. Brucella infection in cirrhotic patients may cause these findings due to more pronounced tissue damage, particularly in elderly patients.

There were several limitations of this study. First, the numbers of patients in the study and control groups were relatively low. Second, the data do not reflect data from all regions. Third, we did not examine the way of contact with Brucella infection due to study design. We postulated that it may have developed through consumption of unpasteurized Turkish herbal cheese. Lastly, due to the retrospective design of the study, we did not perform the treatment outcomes according to Brucella disease activity. It would be useful to understand the impact of Brucella infection on the HDV infected population in terms of symptoms, clinical meaning and outcome, and to determine whether treatment for brucellosis can change their outcome.
In sum, patients with CHD-C from endemic areas of the world are at higher risk of Brucella infection, and high clinical suspicion is needed for diagnosis of acute brucellosis.

In conclusion, brucellosis incidence is extraordinarily high among delta hepatitis related cirrhosis, especially in patients with higher MELD scores. Targeted preventive efforts may decrease the rate of Brucella infection, and Wright testing in highrisk populations can identify individuals in the earliest stages of infection.

\section{Conflict of interest}

The authors declare no conflict of interest.

\section{References}

1. Hughes SA, Wedemeyer H, Harrison PM. Hepatitis delta virus. Lancet 2011; 378: 73-85.

2. Gomaa NI, Metwally LA, Nemr N, Younis S. Seroprevalence of HDV infection in HBsAg positive population in Ismailia, Egypt. Egypt J Immunol 2013; 20: 23-8.

3. Bahcecioglu IH, Aygun C, Gozel N, Poyrazoglu OK, Bulut Y, Yalniz M. Prevalence of hepatitis delta virus (HDV) infection in chronic hepatitis B patients in eastern Turkey: still a serious problem to consider. J Viral Hepat 2011; 18: 518-24.

4. Turkdoğan MK, Bozkurt H, Uygan I, et al. Chronic hepatitis delta virus infection in Van region of eastern Turkey. Turk J Gastroenterol 2005; 16: 17-20.

5. Pappas G, Akritidis N, Bosilkovski M, Tsianos E. Brucellosis. N Engl J Med 2005; 352: 2325-36.

6. Liu Q, Cao L, Zhu XQ. Major emerging and re-emerging zoonoses in China: a matter of global health and socioeconomic development for 1.3 billion. Int J Infect Dis 2014; 25: 65-72.

7. Guler S, Kokoglu OF, Ucmak H, Gul M, Ozden S, Ozkan F. Human brucellosis in Turkey: different clinical presentations. J Infect Dev Ctries 2014; 8: 581-8.

8. Fernández J, Gustot T. Management of bacterial infections in cirrhosis. J Hepatol 2012; 56 Suppl 1: S1-12.

9. Casao MA, Navarro E, Solera J. Evaluation of Brucellacapt for the diagnosis of human brucellosis. J Infect 2004; 49: 102-8.

10. Dienstag JL. Hepatitis B virus infection. N Engl J Med 2008; 359: 1486-500.

11. Heller T, Rotman Y, Koh C, et al. Long-term therapy of chronic delta hepatitis with peginterferon alfa. Aliment Pharmacol Ther 2014; 40: 93-104.

12. Mese S, Nergiz S, Tekes S, Gul K. Seroprevalence of serum HBsAg positivity and hepatitis delta virus infection among blood donors in Southeastern Turkey. Clin Ter 2014; 165: 95-8.

13. Corbel MJ. Microbiological aspects. In: Madkour's Brucellosis. Madkour MM (ed.). Springer-Verlag, New York 2001; 51-64.

14. Turkdogan MK, Akdeniz H, Berktaş M. Evaluation of hepatic involvement in brucellosis. East J Med 1996; 1: 8-9.

15. Kose S, Smits HL, Abdoel TH, Ozbel Y. Prevalence of Brucella antibodies in rural and suburban communities in three provinces of Turkey: need for improved diagnosis and prevention. J Infect 2006; 53: 308-14.

16. Christou L, Pappas G, Falagas ME. Bacterial infection-related morbidity and mortality in cirrhosis. Am J Gastroenterol 2007; 102: 1510-7. 
17. Baijal R, Amarapurkar D, Praveen Kumar HR, et al. A multicenter prospective study of infections related morbidity and mortality in cirrhosis of liver. Indian J Gastroenterol 2014; 33: 336-42.

18. Ferreira AO, Martins LN, Marinho RT, Velosa J. Spontaneous bacterial peritonitis by Brucella in a cirrhotic patient. BMJ Case Rep 2013; 2013: pii: bcr2013008629. doi: 10.1136/bcr-2013-008629.

19. Jacob NR, Rodríguez CG, Binaghi MA, et al. Brucellosis complicating chronic non-infectious disorders: diagnostic and therapeutic dilemmas. J Med Microbiol 2008; 57 : 1161-6.

20. Dizbay M, Hizel K, Kilic S, Mutluay R, Ozkan Y, Karakan T. Brucella peritonitis and leucocytoclastic vasculitis due to Brucella melitensis. Braz J Infect Dis 2007; 11: 443-4.

21. Kaya O, Avsar K, Zeynep Akcam F. Unusual manifestations of brucellosis. Arch Med 2011; 7: 173-5.

22. Al-Shaar L, Chaaya M, Ghosn N, Mahfoud Z. Brucellosis outbreak in Chouf district of Lebanon in 2009 a case-control study. East Mediterr Health J 2014; 20: 250-6.

23. Rahil Al, Othman M, Ibrahim W, Mohamed MY. Brucellosis in Qatar: a retrospective cohort study. Qatar Med J 2014; 2014: 25-30.

24. Esmaeili S, Pourhossein B, Gouya MM, Amiri FB, Mostafavi E. Seroepidemiological survey of $Q$ fever and brucellosis in Kurdistan Province, western Iran. Vector Borne Zoonotic Dis 2014; 14: 41-5. 